\title{
Comparative Study of Human Hematopoietic Cell Engraftment into Balb/c and C57BL/6 Strain of Rag-2/Jak3 Double-Deficient Mice
}

\author{
Ayumi Ono, Shinichiro Hattori, Ryusho Kariya, Sumako Iwanaga, Manabu Taura, \\ Hideki Harada, Shinya Suzu, and Seiji Okada
}

Division of Hematopoiesis, Center for AIDS Research, Kumamoto University, Honjo 2-2-1, Kumamoto 860-0811, Japan

Correspondence should be addressed to Seiji Okada, okadas@kumamoto-u.ac.jp

Received 3 November 2010; Accepted 1 January 2011

Academic Editor: Nafees Ahmad

Copyright (C) 2011 Ayumi Ono et al. This is an open access article distributed under the Creative Commons Attribution License, which permits unrestricted use, distribution, and reproduction in any medium, provided the original work is properly cited.

\begin{abstract}
Immunodeficient mice are becoming invaluable tools in human stem cell and tumor research. In this study, we generated Rag-2/Jak3 double-deficient (Rag- $2^{-1-} \mathrm{Jak}^{-/-}$) mice with a C57/BL6 and Balb/c genetic background and compared the human lymphohematopoietic cell engraftment rate. Human cord blood-derived CD $34^{+}$hematopoietic stem cells were successfully

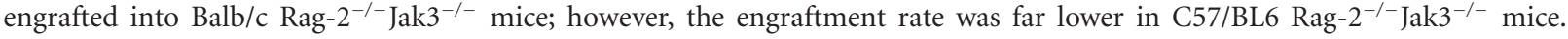
Transplantation of human peripheral blood mononuclear cells resulted in the same tendency. Thus, a Balb/c background offers superior engraftment capacity than a C57/BL6 background and provides an attractive model for human hematopoietic cell engraftment.
\end{abstract}

\section{Introduction}

Animal models with a human hematopoietic and immune system are now important tools for studying the human immune system as well as human-specific infections, such as HIV-1 [1, 2]. Recent approaches have involved the use of genetically immunodeficient mice that have been reconstituted using human-derived hematopoietic stem cells (HSCs) (known as humanized mice). Several immunodeficient mice have been developed and NOD/Scid mice with NK defective genetically modified mice are mainly used for this approach [3-5], because they exhibit deficiencies in macrophage and dendritic cell function, and complement as well as $\mathrm{T}$ and B lymphocyte deficiencies [6]. Rag- $2^{-/-} \gamma \mathrm{c}^{-/-}$mice are also commonly used for establishing humanized mice $[7,8]$, and it is known that the genetic background of Rag$2^{-/-} \gamma \mathrm{c}^{-/-}$mice is influenced by the efficiency of human cell engraftment.

JAK3 is a non-receptor-type tyrosine kinase crucial for mediating signaling from the common $\gamma$-chain of cytokine receptors, and Jak3-deficient mice and common $\gamma$-chain- deficient mice show the same phenotype [9]. Based on these findings, we generated Rag- $2^{-/-}$Jak3 $3^{-/-}$mice with C57/BL6 and $\mathrm{Balb} / \mathrm{c}$ genetic backgrounds. These mice showed a lack of mature T and B lymphocytes and NK cells. Balb/c Rag$2^{-/-} \mathrm{Jak}^{-/-}$mice showed high efficiency of both human $\mathrm{CD} 4^{+}$hematopoietic stem cell (HSC) and peripheral blood mononuclear cell (PBMC) transplantation compared with C57/BL6 Rag-2-/-Jak3 ${ }^{-/-}$mice. Our data show that the genetic background of the mice influences human cell engraftment and Balb/c Rag- $2^{-/-} \mathrm{Jak} 3^{-/-}$mice are becoming an alternative source of humanized mice.

\section{Materials and Methods}

2.1. Mice. C57/BL6 Rag-2/Jak3 double-deficient mice were established by crossing Rag-2-deficient mice [10] (obtained from the Center for Animal Resources and Development, Kumamoto University, Japan) and Jak3-deficient mice [11] (kindly provided by Dr. Takashi Saito). Balb/c Rag-2-deficient (Rag-2 ${ }^{-/-}$) mice and Balb/c Jak3-deficient 
$\left(\mathrm{Jak} 3^{-/-}\right)$mice were established by crossing $\mathrm{Rag}-2^{-/-}$mice or $\mathrm{Jak}^{-/-}$mice with the $\mathrm{Balb} / \mathrm{c}$ strain for 10 generations, respectively. Balb/c Rag-2/Jak3 double-deficient (Rag$2^{-/-} \mathrm{Jak}^{-/-}$) mice were established by crossing Balb/c Rag$2^{-/-}$mice and Balb/c Jak3 ${ }^{-/-}$mice, and were housed and monitored in our animal research facility according to institutional guidelines. All experimental procedures and protocols were approved by the Institutional Animal Care and Use Committee at Kumamoto University.

2.2. Cell Preparation and Transplantation. Peripheral blood and umbilical cord blood samples were collected after obtaining written informed consent and approval from the Ethics Committee of Kumamoto University Faculty of Medical and Pharmaceutical Sciences. CD $34^{+}$cells were isolated using the CD34 Progenitor Cell Isolation Kit (Miltenyi Biotec, Sunnyvale, CA) and transplanted into the liver of irradiated $(4.0 \mathrm{~Gy})$ newborn mice. At 12 weeks after transplantation, peripheral blood was obtained and analyzed by flow cytometry.

Peripheral blood mononuclear cells $\left(2 \times 10^{7}\right.$ cells $)$ were transplanted into 5.0 Gy irradiated adult mice (8-10 weeks old) as described [5]. At 2 weeks after transplantation, peripheral blood was harvested and analyzed by flow cytometry.

2.3. Flow Cytometry. Mouse spleen cells were stained with DX5-FITC (pan NK marker), mCD122 (IL-2R $\beta$ )-PE, mCD19-APC, and mCD3-PE/Cy7 (eBiosciences, San Diego, CA) to detect murine lymphocytes. Peripheral blood samples were obtained from the retro-orbital sinus of transplanted mice, and red blood cells were lysed with an ammonium chloride-potassium buffer and stained with antimouse CD45-APC/Cy7 and antihuman CD45-PB (Biolegends, San Diego, CA). Cells were washed and resuspended in PBS supplemented with $3 \%$ FCS, $0.02 \%$ NaN3, and $1 \mu \mathrm{g} / \mathrm{mL}$ propidium iodide and were analyzed using LSR II (BD Biosciences, San Jose, CA). Data were analyzed with FlowJo (Tree Star, San Carlos, CA).

2.4. Engraftment of K562 Cells. K562 cells (obtained from RIKEN cell bank, Tsukuba, Japan) were cultured in RPMI1640 medium (Sigma) supplemented with 10\% FBS, and were transplanted subcutaneously into the right flank of C57/BL6 Rag-2 $2^{-/-}$Jak3 ${ }^{-/-}$mice and Balb/c Rag-2 $2^{-/-}$Jak3 ${ }^{-/-}$ mice. The tumors were removed and weighed 21 days after transplantation.

2.5. Statistical Analysis. The statistical significance of differences was determined using Student's $t$-test. $P$ values less than .05 were defined as significant.

\section{Results}

3.1. Characterization of Rag- $2^{-/-} \mathrm{Jak}^{-/-}$Mice. The generated C57/BL6 Rag-2 ${ }^{-/-} \mathrm{Jak}^{-/-}$mice and Balb/c Rag$2^{-/-} \mathrm{Jak}^{-/-}$mice survived and bred well under the specific pathogen-free conditions. To confirm the predicted immunophenotype of C57/BL6 Rag-2 $2^{-/-}$Jak3 $3^{-/-}$mice and $\mathrm{Balb} / \mathrm{c}$ Rag-2 ${ }^{-/-} \mathrm{Jak} 3^{-/-}$mice, single-cell suspensions from spleen cells were labeled with fluorescent antibodies against mouse DX-5 (pan NK marker), CD122 (IL-2R $\beta$ ), CD3 (Tcell marker), and CD19 (B-cell marker). In contrast to wildtype mice, no $\mathrm{B}$ (CD20 positive) and $\mathrm{T}$ (CD3 positive) lymphocytes or NK cells (DX-5 and CD122 double-positive cells) were detected in either C57/BL6 or Balb/c Rag$2^{-/-} \mathrm{Jak} 3^{-/-}$mice (Figure 1 ).

3.2. Engraftment with Human Hematopoietic Stem Cells. To determine the engraftment efficiency of human hematopoietic stem cells, $2 \times 10^{5}$ cord blood-derived CD34 ${ }^{+}$cells from normal volunteers were injected intrahepatically into C57/BL6 Rag- $2^{-/-} \mathrm{Jak} 3^{-/-}$mice and Balb/c Rag- $2^{-/-} \mathrm{Jak} 3^{-/-}$ mice as described [5, 7]. Levels of human cell engraftment were determined 12 weeks later (Figure 2(a)). Of the engrafted C57/BL6 Rag-2-/- Jak3 ${ }^{-/-}$mice, 18 of 28 mice had less than $0.1 \%$ of human CD $45^{+}$cells, and only 2 had $>10 \%$ human $\mathrm{CD}_{4} 5^{+}$cells in the peripheral blood $(1.7 \pm 4.5 \%, n=$ 28). In contrast, Balb/c Rag- $2^{-/-} \mathrm{Jak} 3^{-/-}$mice were highly engrafted and reconstituted with human cells $(37.8 \pm 21.5 \%$, $n=24$ ) (Figure 2(b)).

3.3. Engraftment with Human PBMC. To determine the engraftment efficiency of human primary lymphocyte populations, $2 \times 10^{7}$ PBMCs from normal volunteers were injected intraperitoneally into C57/BL6 $\mathrm{Rag}-2^{-/-} \mathrm{Jak} 3^{-/-}$ mice and Balb/c Rag- $2^{-/-} \mathrm{Jak} 3^{-/-}$mice. Levels of human cell engraftment were determined 2 weeks later. Although human cells (human CD $45^{+}$cells) were detected in C57/BL6 Rag- $2^{-/-} \mathrm{Jak}^{-/-}$mice, the degree of engraftment was very low $(1.7 \pm 4.5 \%, n=13)$. In contrast, Balb/c Rag- $2^{-/-} \mathrm{Jak} 3^{-/-}$ mice were highly engrafted with human cells $(37.8 \pm 21.5 \%$, $n=12$ ) (Figure 3).

3.4. Tumor Cell Engraftment. The ability of Rag-2-/- Jak3 $3^{-/-}$ mice to engraft human hematopoietic malignancies was examined. Subcutaneous administration of K562 cells resulted in solid tumor formation in both strains of Rag$2^{-/-} \mathrm{Jak}^{-/-}$mice. The mice were sacrificed and subcutaneous tumors were removed and weighed. The weight of tumors in both strains showed no significant difference (C57/BL6: $0.59 \pm 0.26 \mathrm{~g}, n=7$, Balb/c $0.63 \pm 0.33 \mathrm{~g}, n=7$, $P>.05)$ (Figure 4).

\section{Discussion}

In this study, we established C57/BL6 and Balb/c strains of $\mathrm{Rag}-2^{-/-} \mathrm{Jak} 3^{-/-}$mice, and showed that Balb/c Rag$2^{-/-} \mathrm{Jak}^{-/-}$mice supported a high level of human lymphohematopoietic cell engraftment, whereas C57/BL6 Rag$2^{-/-} \mathrm{Jak}^{-/-}$mice achieved little engraftment. Interestingly, both strains supported the engraftment of human hematopoietic malignancy.

Humanized mice with reconstituted human hematopoietic and immune cells are becoming a powerful tool for the investigation of human biological systems and for 

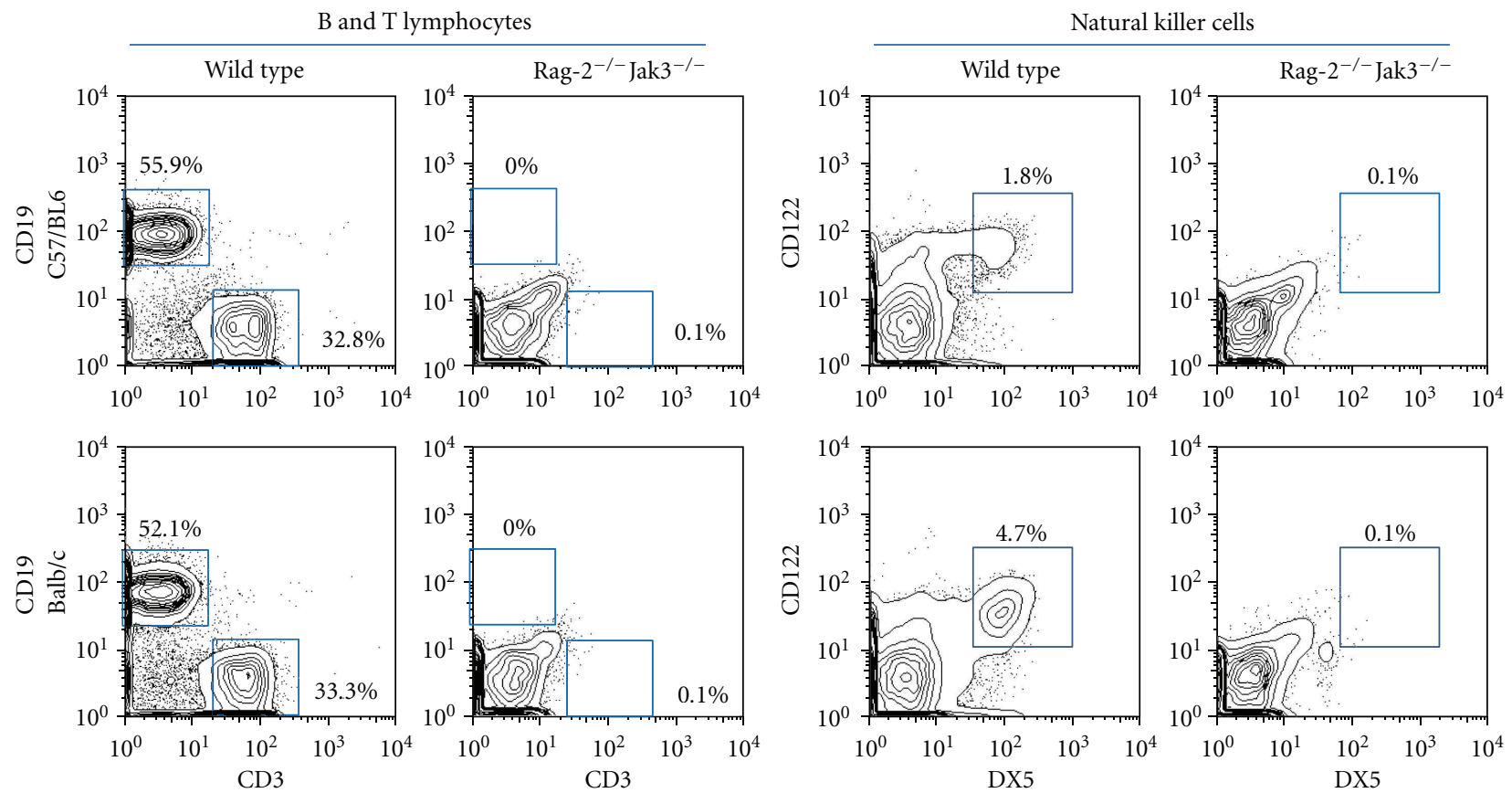

FIgURE 1: Lack of mature lymphocytes and NK cells in both Balb/c and C57/BL6 background Rag-2 $2^{-/-}$Jak3 ${ }^{-/-}$mice. Spleen cells from C57/BL6 wild-type mice, Rag-2 ${ }^{-/-} \mathrm{Jak}^{-/-}$mice and Balb/c wild-type mice, and Rag-2 ${ }^{-{ }^{-}} \mathrm{Jak}^{-/-}$mice, were stained with DX5-FITC (pan NK marker), CD122 (IL-2R $\beta$ ), CD19-APC, and CD3-PE/Cy7. No T and B lymphocytes or NK cells were observed in the spleen of Rag$2^{-/-} \mathrm{Jak}^{-1-}$ mice.

translational research [2]. NOD/Scid mice have been used for these purposes; however, human hematopoietic stem cell engraftment is not sufficient and functionally mature lymphocytes are not developed in these mice, mostly due to residual NK activity in this mouse. To overcome these limitations, NOD/Scid mice were intercrossed with NK defective genetically modified mice, and several strains of mice suitable for humanized mice were established [35]. Among them, NOD/Scid $/ \gamma \mathrm{c}^{-/-}$(NOG and NSG) mice are becoming the gold standard for generating humanized mice. Recently, Spira gene polymorphism has been identified as a determinant influencing the efficacy of human hematopoietic stem cell engraftment into the NOD/Scid strain [12]. Meanwhile, Stroma cells of the Balb/c strain have been shown to support human hematopoiesis to a lesser extent but not other strains (C57/BL6, C3H, ICR), indicating that other mechanisms support human cell engraftment in immunodeficient mice than Spira gene polymorphism. In fact, Manz and colleagues described the reconstitution of human acquired and innate immunity in Balb/c Rag$2^{-/-} \gamma \mathrm{c}^{-/-}$mice [7]. Although no study has compared reconstitution efficiency directly between $\mathrm{NOD} / \mathrm{Scid} / \mathrm{\gamma c}^{-/-}$ mice and Balb/c Rag- $2^{-/-} \gamma \mathrm{c}^{-/-}$mice, the efficiency of human hematopoietic stem cell engraftment seems to be higher in NOD background from the previous studies $[1,2]$. However, the efficiency of reconstitution in Balb/c Rag- $2^{-/-} \gamma \mathrm{c}^{-/-}$mice is high enough for human immune and hematopoietic cell research (Figures 2 and 3) and $\mathrm{Balb} / \mathrm{c}$ background has several advantages compared with NOD/Scid background.
The NOD/Scid strain has several disadvantages [1]. Mice with the scid mutation lack functional $\mathrm{T}$ and $\mathrm{B}$ lymphocytes resulting from the absence of gene recombination events in T-cell receptors (TCRs) and immunoglobulins; however, they are known to show a leaky phenomenon in which functional $\mathrm{T}$ and $\mathrm{B}$ lymphocytes are produced with aging or ionized irradiation $[13,14]$. In addition, the Prkdc gene disrupted by the scid mutation is expressed broadly and is involved in DNA repair, while expressions of Rag-1 and Rag2 are limited to hematopoietic cells and are involved only in DNA recombination of T- and B-cell receptor genes, indicating that scid mice are more sensitive to radiation-induced DNA damage than their Rag- $1^{-/-}$or Rag- $2^{-/-}$counterparts. Recently, T. Pearson et al. established radioresistant NOD Rag- $1^{-/-} \gamma \mathrm{c}^{-/-}$mice and showed that they support a similar level of human lymphohematopoietic cell engraftment [15], which could overcome the disadvantages of the scid mutation. The NOD/Scid strain has a predisposition to thymic lymphoma due to an endogenous ectopic provirus (Emv-30) [16]. NOD/Scid and NOD/Scid/ $\beta 2 \mathrm{~m}^{-/-}$mice have a short mean life span for this reason $[6,17]$, while NOD/Scid $/ \gamma \mathrm{c}^{-/-}$ (NOG and NSG) mice do not develop thymic lymphoma for an unknown reason [4]. Balb/c strain could overcome these disadvantages of NOD/Scid strain, although efficiency of human hematopoietic stem cell engraftment is not better than NOD/Scid strain[1].

As the strain background is known to affect human-cell engraftment and to function markedly in scid mice $[1,2,18]$, we established and directly compared C57/BL6 and Balb/c

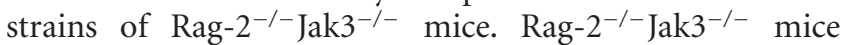



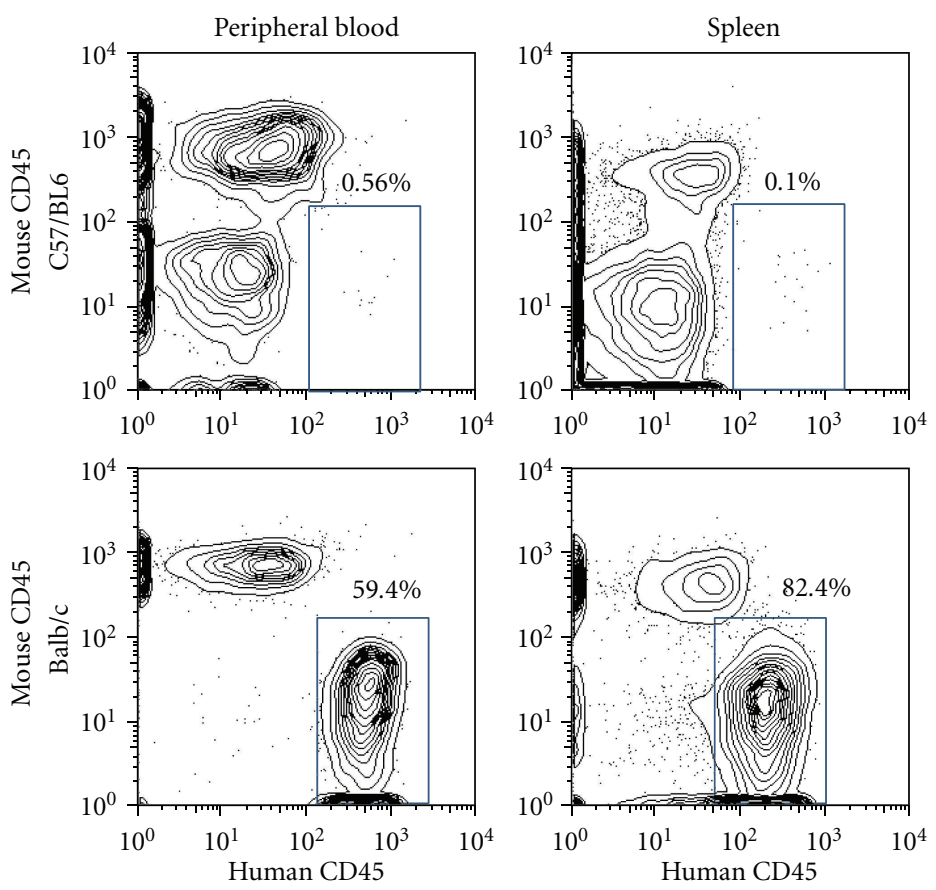

(a)
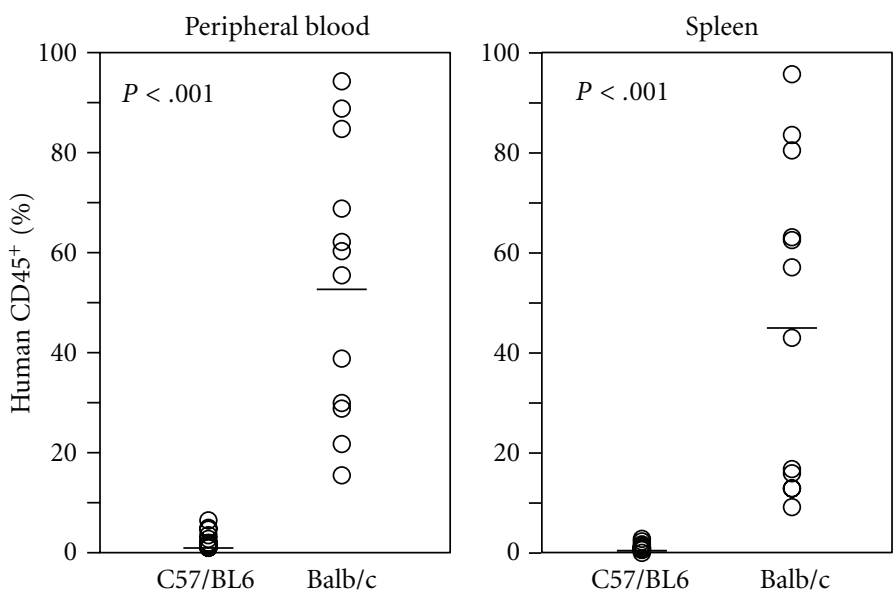

(b)

FIGURE 2: Balb/c Rag-2 $2^{-/-} \mathrm{Jak} 3^{-/-}$mice support human hematopoietic stem cell engraftment more efficiently than C57/BL6 Rag-2 ${ }^{-/-}$Jak3 ${ }^{-/-}$ mice. Cord blood-derived CD34 ${ }^{+}$cells $\left(2 \times 10^{5}\right.$ cells/ mouse $)$ were transplanted into irradiated newborn mice intrahepatically. Peripheral blood of CD34-transplanted recipients was obtained 12 weeks after transplantation, and human and murine chimerism was assessed by flow cytometry.

showed the same phenotype as Rag- $2^{-/-} \gamma \mathrm{c}^{-/-}$mice lacking T, B, and NK cells [7] (Figure 1). The efficiency of human hematopoietic stem cell and PBMC engraftment in C57/BL6 Rag-2 $2^{-/-} \mathrm{Jak}^{-/-}$mice was extremely poor, although Balb/c mice and Rag- $2^{-/-} \mathrm{Jak}^{-/-}$mice appeared to have similar levels as Balb/c Rag- $2^{-/-} \gamma \mathrm{c}^{-/-}$mice (Figures 2 and 3) [8]. The breeding performance of Balb/c Rag- $2^{-/-} \gamma \mathrm{c}^{-/-}$mice was the same as Balb/c mice in our study. In addition, Balb/c Rag- $2^{-/-} \gamma \mathrm{c}^{-/-}$mice were relatively radioresistant compared with NOD/Scid strains. Thus, Balb/c Rag- $2^{-/-} \mathrm{Jak} 3^{-/-}$mice are a very useful strain of immunodeficient mice derived from animals with defined molecular lesions and have distinct advantages over NOD/Scid-based immunodeficient mice.

\section{Conclusion}

In conclusion, we established C57/BL6 and Balb/c strains of $\mathrm{Rag}^{-2^{-/}} \mathrm{Jak3}^{-/-}$mice and showed that Balb/c Rag$2^{-/-} \mathrm{Jak}^{-/-}$mice enabled high human hematopoietic cell engraftment. 

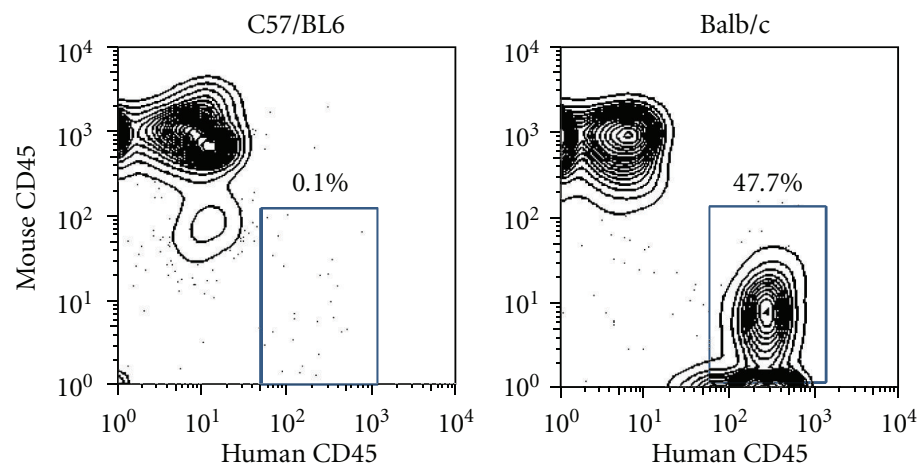

(a)

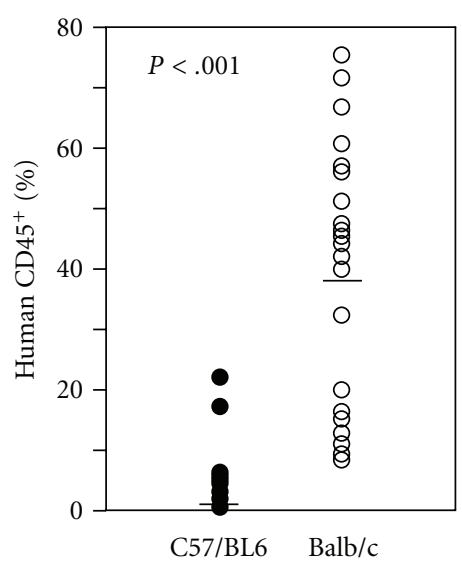

(b)

FIGURE 3: Balb/c Rag-2-/- Jak3-/- mice support human peripheral blood mononuclear cells (PBMC) engraftment more efficiently than C57/BL6 Rag- $2^{-/-} \mathrm{Jak3}^{-/-}$mice. PBMC $\left(2 \times 10^{7}\right.$ cells/mouse $)$ were transplanted into irradiated adult mice intravenously. Peripheral blood of PBMC transplanted recipients were obtained 12 weeks after transplantation, and human and murine chimerism was assessed by flow cytometry.

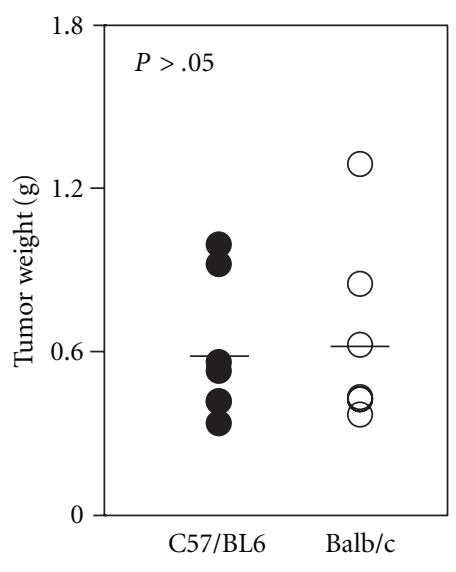

Figure 4: Engraftment of K562 erythroleukemia cell line into Rag- $2^{-/-}$Jak3 ${ }^{-1-}$ mice. K562 cell line $\left(5 \times 10^{6}\right.$ cells/ mouse $)$ was subcutaneously transplanted into $\mathrm{Rag}-2^{-/-} \mathrm{Jak} 3^{-/-}$mice. Three weeks after transplantation, mice were sacrificed and the tumors weighed.

\section{Acknowledgments}

The authors are grateful to Dr. Matsui and his coworkers (Fukuda Hospital, Kumamoto, Japan) for providing human umbilical cord blood, Dr. T. Saito for providing Jak3deficient mice, Ms. Y. Endo and K. Tokunaga for secretarial assistance, and Ms. I. Suzu and Ms. Y. Fukuya for skillful technical assistance. This work was supported in part by Health and Labour Sciences Research Grants from the Ministry of Health, Labour and Welfare of Japan (no. H22-AIDSI-002) and by the Global COE program "Global Education and Research Center Aiming at the Control of AIDS" and Grants-in-Aid for Science Research (nos. 21107522 and 21591209) from the Ministry of Education, Sports, Culture, Science, and Technology of Japan.

\section{References}

[1] N. Legrand, K. Weijer, and H. Spits, "Experimental models to study development and function of the human immune 
system in vivo," Journal of Immunology, vol. 176, no. 4, pp. 2053-2058, 2006.

[2] L. D. Shultz, F. Ishikawa, and D. L. Greiner, "Humanized mice in translational biomedical research," Nature Reviews Immunology, vol. 7, no. 2, pp. 118-130, 2007.

[3] T. Yahata, K. Ando, Y. Nakamura et al., "Functional human $\mathrm{T}$ lymphocyte development from cord blood CD34+ cells in nonobese diabetic/Shi-scid, IL-2 receptor gamma null mice," Journal of Immunology, vol. 169, no. 1, pp. 204-209, 2002.

[4] F. Ishikawa, M. Yasukawa, B. Lyons et al., "Development of functional human blood and immune systems in NOD/SCID/IL2 receptor $\gamma$ chain mice," Blood, vol. 106, no. 5, pp. 1565-1573, 2005.

[5] S. Okada, H. Harada, T. Ito, T. Saito, and S. Suzu, "Early development of human hematopoietic and acquired immune systems in new born NOD/Scid/Jak3 mice intrahepatic engrafted with cord blood-derived CD34+ cells," International Journal of Hematology, vol. 88, no. 5, pp. 476-482, 2009.

[6] L. D. Shultz, P. A. Schweitzer, S. W. Christianson et al., "Multiple defects in innate and adaptive immunologic function in NOD/LtSz-scid mice," Journal of Immunology, vol. 154, no. 1, pp. 180-191, 1995.

[7] E. Traggiai, L. Chicha, L. Mazzucchelli et al., "Development of a human adaptive immune system in cord blood celltransplanted mice," Science, vol. 304, no. 5667, pp. 104-107, 2004.

[8] J. P. Goldman, M. P. Blundell, L. Lopes, C. Kinnon, J. P. Di Santo, and A. J. Thrasher, "Enhanced human cell engraftment in mice deficient in RAG2 and the common cytokine receptor $\gamma$ chain," British Journal of Haematology, vol. 103, no. 2, pp. 335-342, 1998.

[9] K. Suzuki, H. Nakajima, Y. Saito, T. Saito, W. J. Leonard, and I. Iwamoto, "Janus kinase 3 (Jak3) is essential for common cytokine receptor $\gamma$ chain $(\gamma(\mathrm{c}))$-dependent signaling: comparative analysis of $\gamma(\mathrm{c})$, Jak3, and $\gamma(\mathrm{c})$ and Jak3 doubledeficient mice," International Immunology, vol. 12, no. 2, pp. 123-132, 2000.

[10] Y. Shinkai, G. Rathbun, K. P. Lam et al., "RAG-2-deficient mice lack mature lymphocytes owing to inability to initiate $\mathrm{V}(\mathrm{D}) \mathrm{J}$ rearrangement," Cell, vol. 68, no. 5, pp. 855-867, 1992.

[11] S. Y. Park, K. Saijo, T. Takahashi et al., "Developmental defects of lymphoid cells in Jak3 kinase-deficient mice," Immunity, vol. 3, no. 6, pp. 771-782, 1995.

[12] K. Takenaka, T. K. Prasolava, J. C. Y. Wang et al., "Polymorphism in Sirpa modulates engraftment of human hematopoietic stem cells," Nature Immunology, vol. 8, no. 12, pp. 13131323, 2007.

[13] J. S. Danska, F. Pflumio, C. J. Williams, O. Huner, J. E. Dick, and C. J. Guidos, "Rescue of T cell-specific V(D)J recombination in SCID mice by DNA-damaging agents," Science, vol. 266, no. 5184, pp. 450-455, 1994.

[14] G. C. Bosma, M. Fried, R. P. Custer, A. Carroll, D. M. Gibson, and M. J. Bosma, "Evidence of functional lymphocytes in some (leaky) scid mice," Journal of Experimental Medicine, vol. 167, no. 3, pp. 1016-1033, 1988.

[15] T. Pearson, L. D. Shultz, D. Miller et al., "Non-obese diabeticrecombination activating gene-1 (NOD-Rag1) interleukin (IL)-2 receptor common gamma chain (IL2r $\gamma$ ) null mice: a radioresistant model for human lymphohaematopoietic engraftment," Clinical and Experimental Immunology, vol. 154, no. 2, pp. 270-284, 2008.
[16] M. Prochazka, H. R. Gaskins, L. D. Shultz, and E. H. Leiter, "The nonobese diabetic scid mouse: model for spontaneous thymomagenesis associated with immunodeficiency," Proceedings of the National Academy of Sciences of the United States of America, vol. 89, no. 8, pp. 3290-3294, 1992.

[17] S. W. Christianson, D. L. Greiner, R. Hesselton et al., "Enhanced human CD4+ T cell engraftment in beta2microglobulin-deficient NOD-scid mice," Journal of Immunology, vol. 158, no. 8, pp. 3578-3586, 1997.

[18] R. M. Hesselton, D. L. Greiner, J. P. Mordes, T. V. Rajah, J. L. Sullivan, and L. D. Shultz, "High levels of human peripheral blood mononuclear cell engraftment and enhanced susceptibility to human immunodeficiency virus type 1 infection in NOD/LtSz-scid/scid mice," Journal of Infectious Diseases, vol. 172, no. 4, pp. 974-982, 1995. 


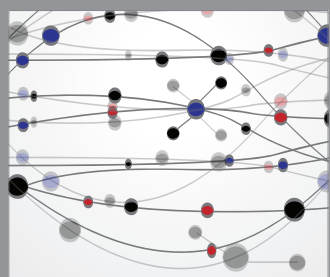

The Scientific World Journal
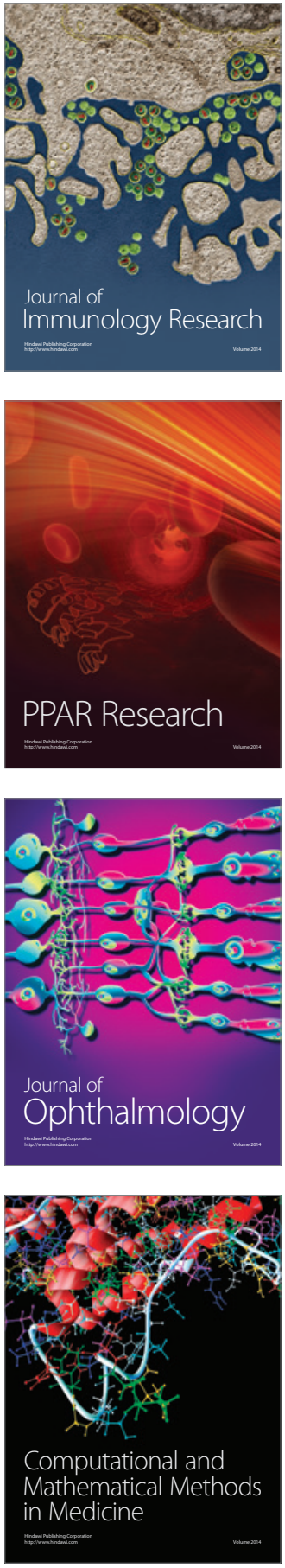

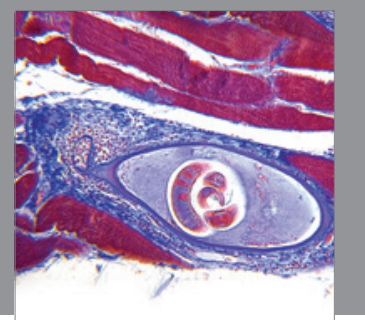

Gastroenterology

Research and Practice
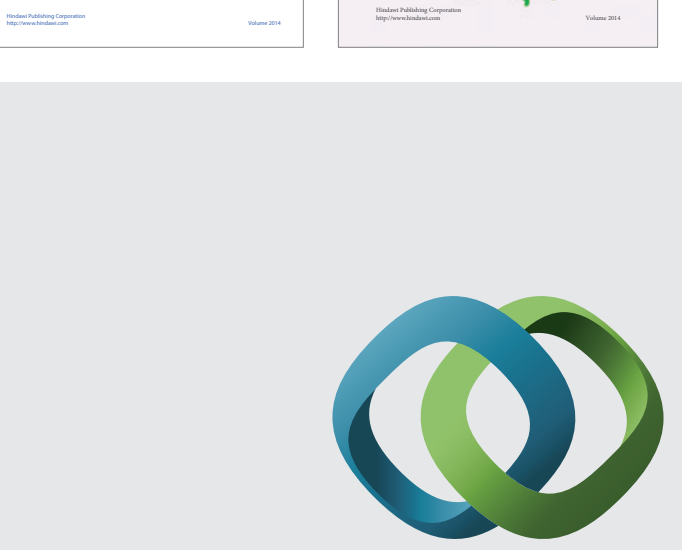

\section{Hindawi}

Submit your manuscripts at

http://www.hindawi.com
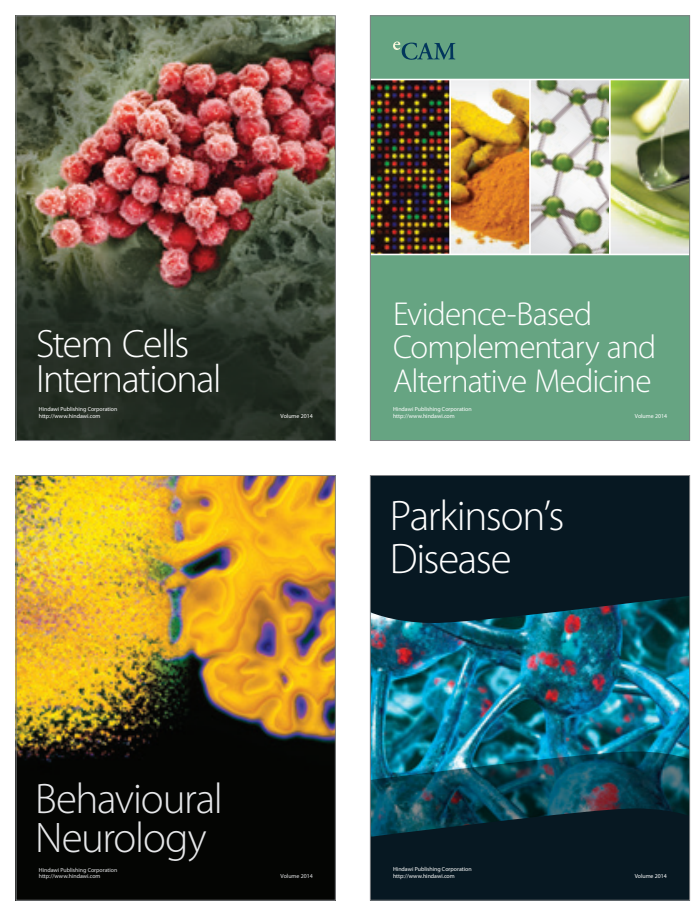

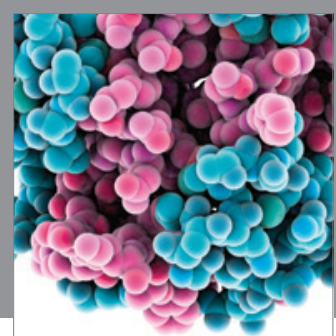

Journal of
Diabetes Research

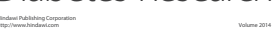

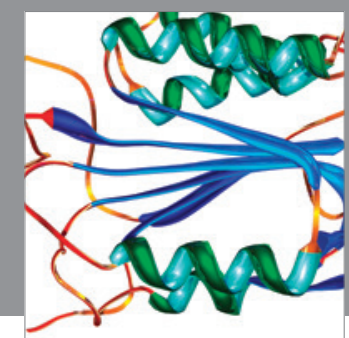

Disease Markers
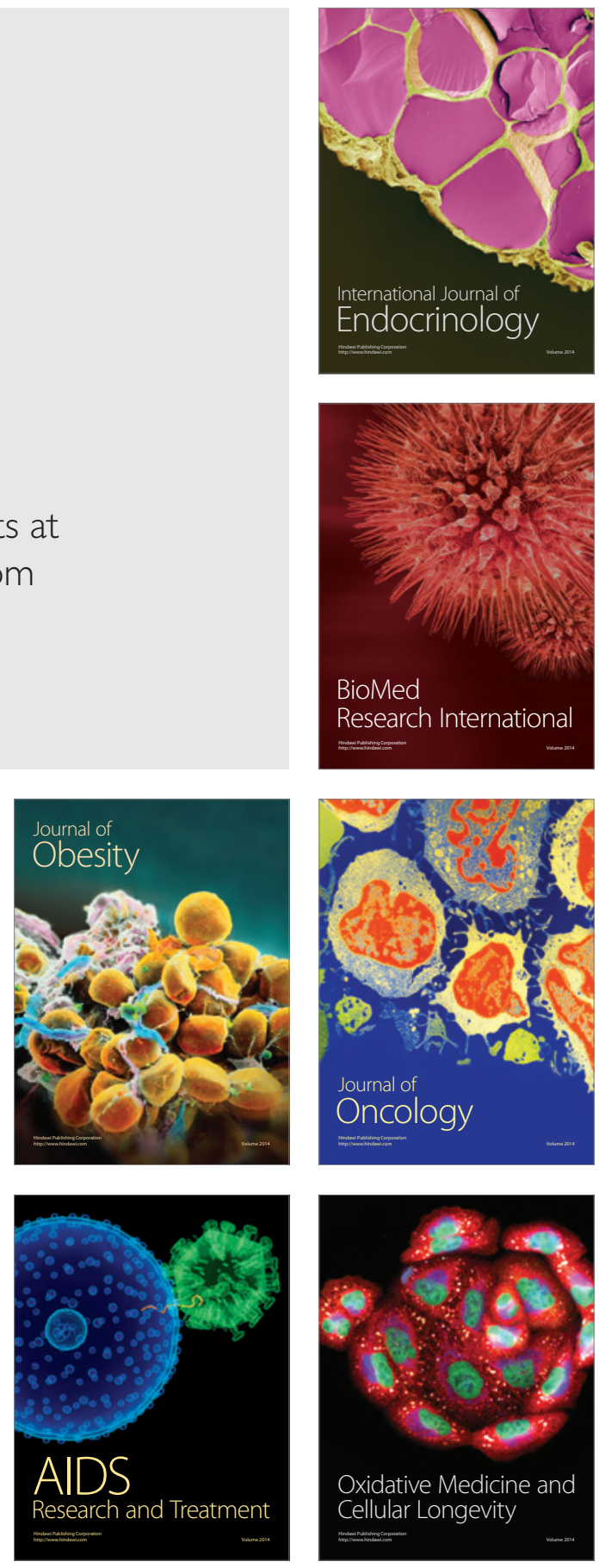